\title{
A Mobile-Based App (MyChoices) to Increase Uptake of HIV Testing and Pre-Exposure Prophylaxis by Young Men Who Have Sex With Men: Protocol for a Pilot Randomized Controlled Trial
}

Katie B Biello ${ }^{1,2,3,4}, \mathrm{PhD}$, MPH; Elliot Marrow ${ }^{3}$, BA; Matthew J Mimiaga ${ }^{1,2,3,4}$, ScD, MPH; Patrick Sullivan ${ }^{5}$, DVM, $\mathrm{PhD}$; Lisa Hightow-Weidman ${ }^{6}$, MD, MPH; Kenneth $\mathrm{H}_{\text {Mayer }}{ }^{3,7,8}$, MD

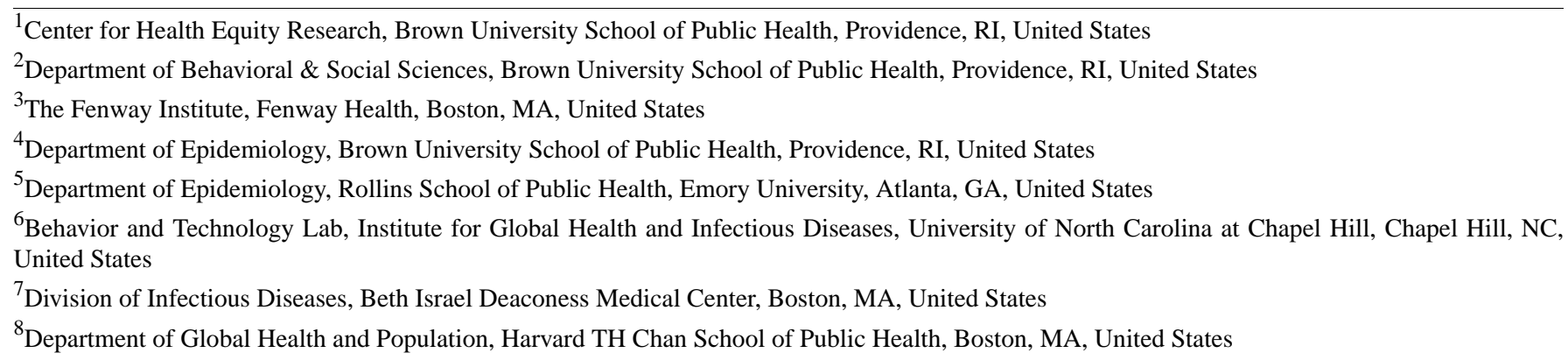

\section{Corresponding Author:}

Katie B Biello, PhD, MPH

Center for Health Equity Research

Brown University School of Public Health

Box G-S121-8

Providence, RI, 02912

United States

Phone: 14018633082

Email: katie biello@brown.edu

\section{Abstract}

Background: HIV incidence is growing most rapidly in the United States among young men who have sex with men (YMSM). Overwhelming evidence demonstrates that routine testing and expanded use of pre-exposure prophylaxis (PrEP) would dramatically reduce the population burden of HIV; however, uptake of both interventions is suboptimal among young adults. The use of mobile phone apps by YMSM is ubiquitous and may offer unique opportunities for public health interventions. MyChoices is a theory-driven app to increase HIV testing and PrEP uptake. It was developed by an interdisciplinary team based on feedback from a diverse sample of YMSM.

Objective: The aim of this paper is to describe the protocol for the refinement, beta testing, and pilot randomized controlled trial (RCT) to examine the acceptability and feasibility of the MyChoices app.

Methods: This 3-phase study includes 4 theater testing groups for app refinement with a total of approximately 30 YMSM; for beta testing, including quantitative assessments and exit interviews, with approximately 15 YMSM over a 2-month period; and for a pilot RCT with 60 YMSM. The pilot will assess feasibility, acceptability, and preliminary efficacy of the MyChoices app, compared with referrals only, in increasing HIV testing and PrEP uptake. All participants will be recruited at iTech clinical research sites in Boston, MA, and Bronx, NY.

Results: App refinement is underway. Enrollment for the pilot RCT began in October 2018.

Conclusions: MyChoices is one of the first comprehensive, theory-driven HIV prevention apps designed specifically for YMSM. If MyChoices demonstrates acceptability and feasibility in this pilot RCT, a multicity, 3-arm randomized controlled efficacy trial of this app and another youth-optimized app (LYNX) versus standard of care is planned within iTech. If shown to be efficacious, the app will be scalable, with the ability to reach YMSM across the United States as well as be geographically individualized, with app content integrated with local prevention and testing activities.

International Registered Report Identifier (IRRID): PRR1-10.2196/10694 
(JMIR Res Protoc 2019;8(1):e10694) doi: 10.2196/10694

\section{KEYWORDS}

adolescents; HIV; men who have sex with men; mHealth; mobile phone; pre-exposure prophylaxis

\section{Introduction}

\section{Background}

In the United States, HIV incidence is growing most rapidly among young men who have sex with men (YMSM). More than $20 \%$ of all new HIV infections in the United States are among young people aged $13-24$ years, and $92 \%$ of these new infections are diagnosed in YMSM, making them one of the largest risk groups for HIV [1]. Men who have sex with men (MSM) of color are disproportionately impacted by the epidemic; in 2015, significantly more black MSM were diagnosed with HIV than white MSM, despite the relatively lower numbers of black MSM overall. HIV diagnoses among young Latino MSM have increased by $14 \%$ [1]. Importantly, it is estimated that compared with the general population, a higher proportion of YMSM (13\% vs $44 \%$, respectively) living with HIV do not know that they are infected [2,3] and, thus, will incur a delay seeking effective treatment, making it more likely that they will transmit HIV to others.

Overwhelming evidence demonstrates that routine testing, resulting in early identification, and therefore, early treatment, of HIV infection, and expanded use of pre-exposure prophylaxis (PrEP) would dramatically reduce the population burden of HIV as well as improve health outcomes for those who are infected [4-8]. However, HIV testing and use of PrEP among young adults is suboptimal. While at least biannual HIV testing is recommended for sexually active MSM, data suggest that approximately $60 \%$ of YMSM do not get even annual HIV tests [9]. Moreover, uptake of PrEP has remained low [10], particularly among young people. In a national Web-based survey of MSM, only 6\% of those aged 18-24 years had ever used PrEP compared with $18 \%$ of those in the $30+$ age group [11].

The normal developmental trajectory of adolescence and young adulthood involves behavioral experimenting, risk taking, and confronting a host of difficult choices with regard to identity formation [12]. These age-appropriate behaviors, beliefs of invincibility, and still-developing cognitive processes may play a role in increasing HIV risk taking behaviors and in placing a low priority on HIV testing and uptake of prevention strategies, particularly PrEP, which requires taking a daily pill to be effective [13].

The ongoing and growing HIV risk for YMSM highlights the need to reach younger individuals using developmentally appropriate, innovative methods and modalities. In addition to expanding access to effective prevention modalities, innovative methods to reach YMSM "where they are" must be developed. Smartphone use is nearly universal among youth in the United States [14]. Younger adults, racial and ethnic minorities, and socioeconomically disadvantaged populations have been identified as having high rates of smartphone use, reducing concerns of inequitable access to the technology [15]. The use of mobile phone apps is ubiquitous and is a common way in which youth interact, get information, and meet sex partners. Mobile apps offer unique opportunities for public health interventions, including efforts to increase HIV testing and PrEP uptake, particularly for YMSM, who may be open to receiving information in a familiar and discreet environment.

Although the popularity of mobile health apps is growing, there are limited data on the efficacy of app-based interventions to enhance HIV prevention and increase HIV testing among MSM, particularly YMSM. However, formative research suggests that Web-based, mobile, or social media outlets are acceptable and feasible means to increase the uptake of prevention services and HIV testing among MSM [16-20]. Informed by extensive formative research, Dr. Patrick Sullivan of Emory University (one of iTech's Principal Investigators), together with app developers from Keymind, developed and tested an HIV testing promotion app for adult MSM (HealthMindr) [21]. Our initial formative research with YMSM [22] suggested interest in the basic functionalities of an HIV testing app like HealthMindr, but the youth felt that it would not be culturally and developmentally appropriate for their peers without further development. As a result, MyChoices incorporates youth feedback to realize an app that draws on the HIV testing functionality of HealthMindr but has been significantly redesigned in the following ways: (1) sexual health information presented in a simple, streamlined, and integrated fashion; (2) designed with attention to having a youth-friendly, social media style appeal; and (3) reduction of text by employing icons, graphics interchange formats (GIFs), and videos.

\section{Theoretical Framework for Intervention}

The MyChoices app is informed by the social cognitive theory (SCT), which specifies goal setting, self-efficacy, and self-regulation as essential influences of health behavior [23,24]. SCT holds that cognition, behavior, and environmental influences interact with one another and reinforce one another to impact health behavior (Figure 1). 
Figure 1. Theoretical model for the MyChoices app development. PrEP: pre-exposure prophylaxis.

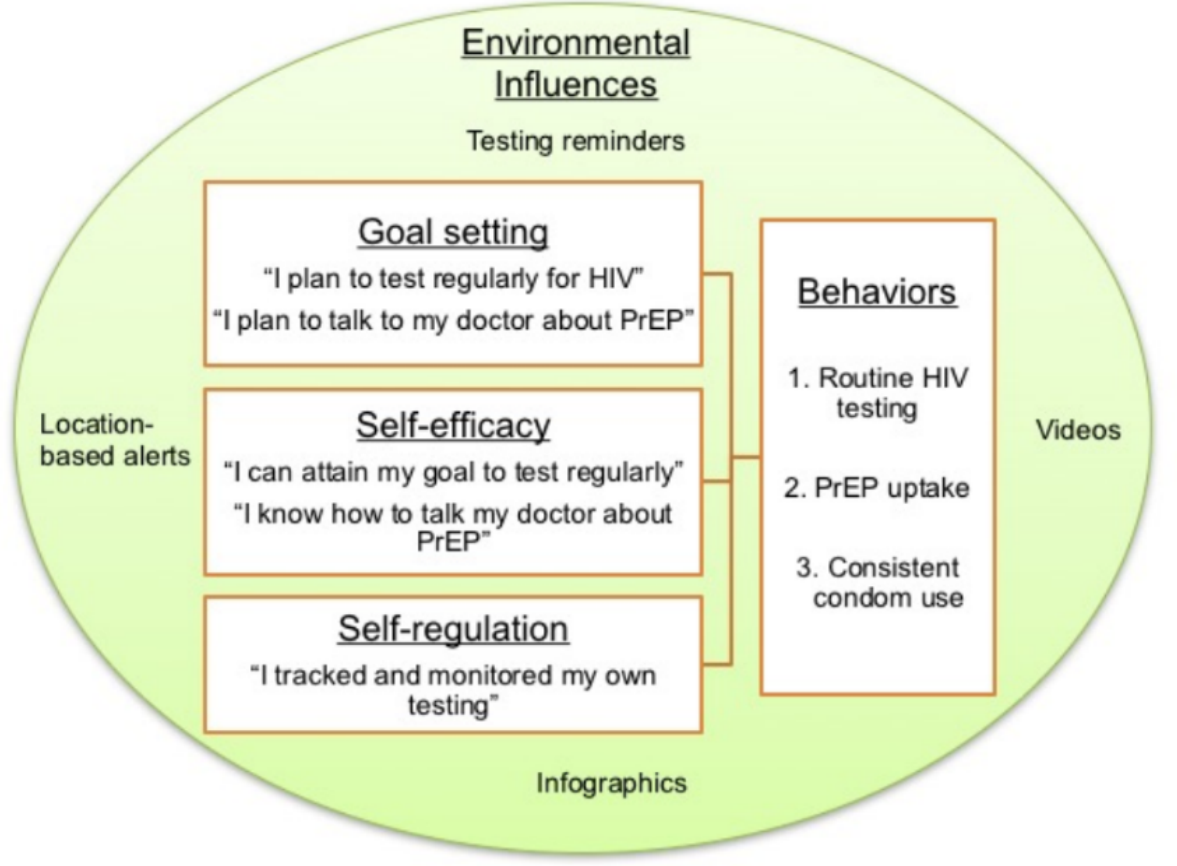

For example, self-regulatory functions (eg, self-monitoring one's HIV testing through development of testing plans) and self-efficacy (eg, belief that one can attain the goal to test regularly) are enhanced by facilitative environmental conditions $[23,24]$ such as reminder systems. MyChoices uses multimedia capabilities and environmental influences to support self-regulation and self-efficacy by enhancing the feeling of control over one's ability to get tested regularly for HIV and use PrEP. Brief surveys about sexual risk and protective health behaviors within the app are used to assist users in tracking and self-monitoring their behaviors and creating a personalized HIV testing plan. Quizzes, videos, and infographics as well as "Help me Choose" (a quiz to help recommend a preferred setting for HIV testing), "Ordering" (a Web-based store to order free condoms, condom-compatible lubricant, and at-home HIV test kits), and geofencing (using global positioning system [GPS] to notify users when they are near a test site and due for testing) functions are used to maximize self-efficacy around HIV prevention and uptake of PrEP.

\section{Aims and Objectives}

The aim of this paper is to describe the protocol for the refinement of an HIV prevention mobile app, beta testing it, and conducting a pilot randomized controlled trial (RCT) to examine the acceptability and feasibility of the MyChoices app for YMSM. We hypothesize that participants who are randomized to use the MyChoices app will report that the app is highly acceptable and will use the main functions of the app over the follow-up period. We also hypothesize that while not powered to detect significant differences, MyChoices users will report taking more HIV tests and increasing PrEP uptake compared with YMSM receiving the control condition.

\section{Methods}

\section{Phase 1: App Refinement}

To refine and enhance the MyChoices app for HIV testing and PrEP uptake among YMSM, we will conduct 4 theater testing groups with approximately 30 YMSM. Testing will take place at 2 iTech subject recruitment venues (SRVs; Boston, MA, and Bronx, NY), and groups will provide suggestions and feedback to improve app acceptability and feasibility as well as approaches to HIV prevention [25]. We aim to have 5-8 participants per group in order to balance the need to have an intimate enough group to share insights, yet large enough to ensure diversity of opinions [26,27]. Participants will be HIV-uninfected YMSM aged between 15 and 24 years who have not had a recent HIV test. For 15-17-year olds, participants must also self-report any anal sex with a male or transfemale partner. For 18-24-year olds, participants must self-report at least one of the following in the past 6 months:

1. $\geq 1$ episode of condomless anal sex with an HIV-positive or unknown HIV status male or transfemale partner

2. Anal sex with $\geq 2$ male or transfemale partners

3. Exchange of money, gifts, shelter, or drugs for anal sex with a male or transfemale partner

4. Sex with a male or transfemale partner and having had a sexually transmitted infection (STI)

5. Sexual partner of an HIV-positive man or transfemale with whom condoms were not consistently used

From our prior experience in recruiting YMSM, we found that it is necessary to include YMSM who engage in lower sexual risk (eg, anal sex with condoms) for the younger age group. Given that risk changes over time, we will still be enrolling an at-risk population who could benefit from the app and who should be testing for HIV regularly. 
Prior to theater testing, participants will complete a brief demographic and behavioral questionnaire in order to contextualize the group data. During theater testing, participants will be asked to interact with the MyChoices app, and feedback will be elicited on the overall appearance and functionality of the app interface, appeal, and usability; ways to maximize acceptability (eg, update language, improve flow, etc); components they like and/or dislike; and areas for improvement. Guided by the SCT model, we will specifically obtain insight into the functionalities and content aimed at impacting self-regulation, self-efficacy, and environmental influences as they relate to HIV prevention.

Theater testing groups will last 60-90 minutes, and discussions will be audiorecorded and transcribed verbatim. Facilitators will also take notes and complete standardized debriefing forms immediately following the visit. These data will allow us to make final refinements to the app, as well as the intervention protocol and assessment battery, prior to initiation of the open technical evaluation and RCT pilot.

\section{Phase 2: Beta Testing}

After refinement as described above, the MyChoices app will undergo beta testing with a small group of YMSM participants (up to 15 YMSM at 2 iTech SRVs: Boston, MA, and Bronx, NY) over a 2-month period. Using the same criteria as Phase 1, participants will be HIV-uninfected young cisgender MSM aged between 15 and 24 years who have not had a recent HIV test and self-report evidence of risk for acquiring HIV infection. For this phase, participants will also be required to own or lease an Android phone, as MyChoices will only be available on an Android platform during beta testing until it is coded by the same developers in iOS for the pilot RCT. This delay is meant to reduce the cost of recoding multiple versions of the edited app. Besides the slight native differences and system-specific limitations between the two platforms, the app was designed in such a way that the visual elements, capabilities, and interactions would be as similar as possible across both platforms.

All participants will be given brief instructions on the purpose of the MyChoices app and an overview of how to use it. Because the primary objective of the beta testing phase is to test instruments and procedures to be used in the pilot RCT, participants will complete the same assessments and processes to be used in the pilot RCT (see pilot RCT section for details). In brief, we will assess acceptability of the MyChoices app using the System Usability Scale (SUS) [28], and feasibility will be assessed using app analytics to determine whether the app was used and what functionalities were most likely and least likely to be opened. Participants will also be asked to provide feedback during Web-based exit interviews conducted by study staff using a Health Insurance Portability and Accountability Act-compliant videoconferencing software (VSee; VSee Lab, LLC, Sunnyvale, CA) technology. Feedback will be requested on functionality, technical performance, errors and software bugs encountered, overall experiences using the app, feedback for further refinement, and subjective impact of the app on HIV testing and PrEP uptake.

A rapid analysis of the data from the exit interviews will be conducted using the detailed notes taken on the debrief forms
[29]. The study team will meet to discuss themes that emerged in the qualitative exit interviews, usage patterns, and acceptability ratings. We will triangulate the findings in order to refine the app, intervention protocol, and assessment tools prior to the pilot RCT [30].

\section{Phase 3: Pilot Randomized Controlled Trial}

\section{Study Design}

A pilot RCT will be conducted at 2 iTech SRVs (Boston, MA, and Bronx, NY) to evaluate the feasibility and acceptability of the MyChoices app and examine the preliminary efficacy of the app in increasing HIV testing and PrEP uptake compared with a standard of care control group. This information will determine whether MyChoices moves forward to be tested in a full-scale efficacy trial planned with iTech. We will enroll 60 YMSM across 2 iTech SRVs who will be randomized to receive either the MyChoices app or standard of care. Participants will be followed for 6 months and will complete a Web-based assessment every 3 months (Figure 2).

\section{Trial Registration, Ethics, Consent, and Institutional Board Approval}

The research and ethics presented in this study have been reviewed and approved by the University of North Carolina at Chapel Hill Institutional Review Board (17-0256). A Certificate of Confidentiality has been obtained from the National Institute of Child Health and Human Development, and a waiver of parental consent will be obtained for participants who are 15-17 years old. The study is also registered on ClinicalTrials.gov (NCT03179319). All participants will undergo screening in a private room at the clinical research site. If eligible (see below), the informed consent discussion will be conducted at this time. The informed consent documents will include detailed information on all study procedures and answer questions concerning the study and assent or consent process.

\section{Participants}

Study participants $(\mathrm{N}=60)$ will be assessed for eligibility by completing a brief screener. Inclusion criteria are identical to those in Phase 2 (beta testing), except that participants must own and be willing to download the app to a phone running either an Android or iOS platform. YMSM of color will be oversampled in order to reach a target enrollment of approximately $50 \%$ and to ensure sufficient representation of the population with the most need.

\section{Recruitment}

Active recruitment will be carried out by study staff at the SRVs by recruiting individuals from organizations and venues where YMSM attend. This may include community-based organizations for sexual and gender minority youth, youth Pride events, etc. Additionally, passive approaches for recruitment will include posting study information at these venues via flyers, posters, and palm cards describing the study. Web-based recruitment will be conducted by placing banner advertisements on popular Web-based social media outlets (eg, Facebook, Grindr, etc). 
Figure 2. The MyChoices pilot randomized controlled trial schema.

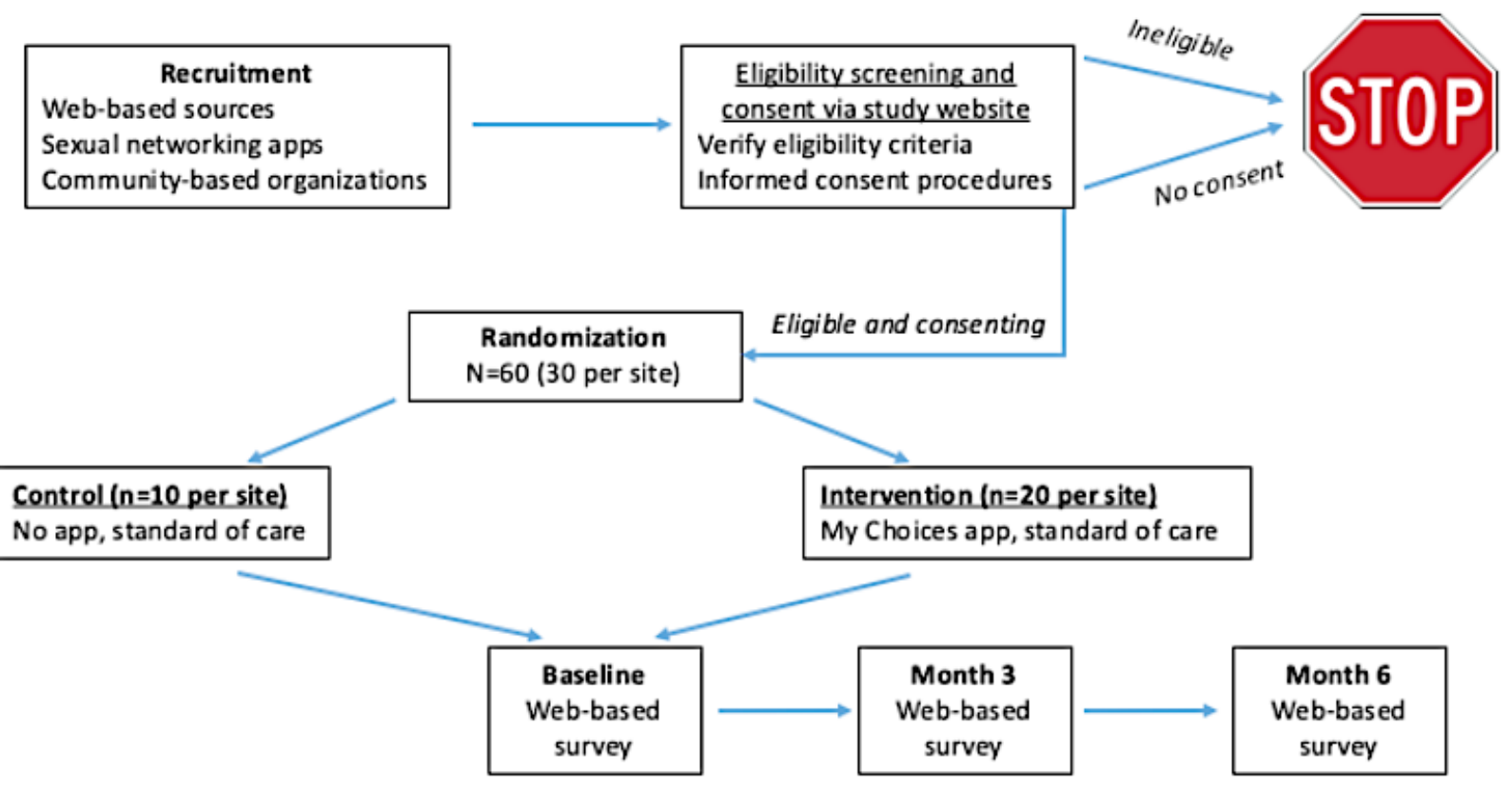

\section{Randomization}

Only participants who express interest in using MyChoices for HIV prevention, meet eligibility criteria, provide informed consent, and complete a baseline assessment will be eligible for randomization (see Figure 2). Randomization will be stratified by SRV [31] and will occur in a 2:1 ratio with 40 YMSM randomized to the experimental condition (20 at each SRV) and 20 randomized to the control condition (10 at each SRV). This allocation will allow us to efficiently gather additional data on app utilization. Randomization will be based on a pregenerated list created by the iTech Analytic Core statisticians, with random blocks of size 3 or 6 , and will be accessed through a Web portal.

Men who are randomized to receive MyChoices will be given brief instructions on the purpose of the app, how to access it, and an overview of how to use it. Participants will be encouraged to explore all components of the app and use it routinely.

\section{Incentives}

Participants in the pilot RCT will receive US \$50 compensation for the in-person screening or baseline assessment and US \$25 compensation for each completed Web-based follow-up assessment.

\section{Intervention}

\section{Standard of Care Condition}

Following completion of the baseline assessment via computer-assisted self-interviewing (CASI), participants in both conditions will receive written prevention material including recommendations for HIV testing and referrals to local HIV testing sites and prevention services.

\section{MyChoices Intervention Condition}

The main functions of the app coincide with the core elements of the SCT of behavior change and are described below (see Figure 3).

\section{Self-Regulation of HIV Risk}

Brief surveys within the app are used to assess individual behavior patterns, particularly around sexual relationships. This information is used to customize the users' app experience. For example, users who report having a main partner will be informed about couples counseling and users who report difficulties using condoms will be provided information about PrEP and links to HIV prevention services at local clinical sites. Users will be asked to complete these brief surveys monthly, which will allow recommended prevention activities to be updated based on recent behaviors. Users are also able to enter HIV and STI test results to keep track of past testing dates and results.

\section{Self-Efficacy for HIV Testing and HIV Prevention}

A number of components target self-efficacy by empowering YMSM to be confident that they can effectively manage engaging in HIV testing, PrEP uptake, and condom use. For example, quizzes, infographics, and GIFs that focus on the promotion of HIV prevention and regular HIV testing and that appeal to YMSM have been incorporated. Links to videos related to PrEP (eg, what is PrEP and talking to your doctor about PrEP), reasons for routine HIV testing, and the importance of engaging in care if one tests positive are included. The MyChoices app also allows users to order home-testing kits for HIV and STIs (syphilis and rectal and urethral gonorrhea and chlamydia) and different types of condoms and lubricants that can be shipped directly to them or to another more confidential location of their choosing via an arrangement with Amazon. Additionally, the app includes information on and links to testing sites and local PrEP clinics (eg, contact number, address, and 
testing hours) as determined using GPS technology, allowing men to locate clinics that are nearby their current location.

Figure 3. The MyChoices home screen.

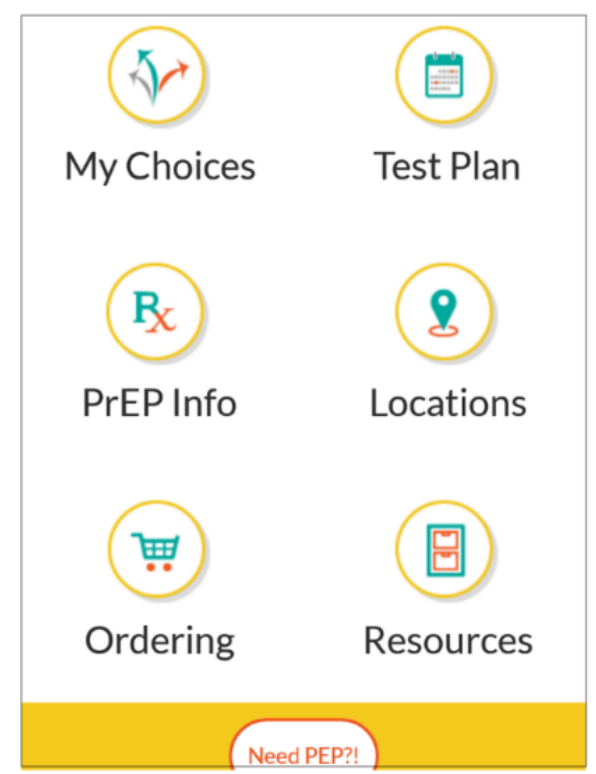

Figure 4. The MyChoices test plan.

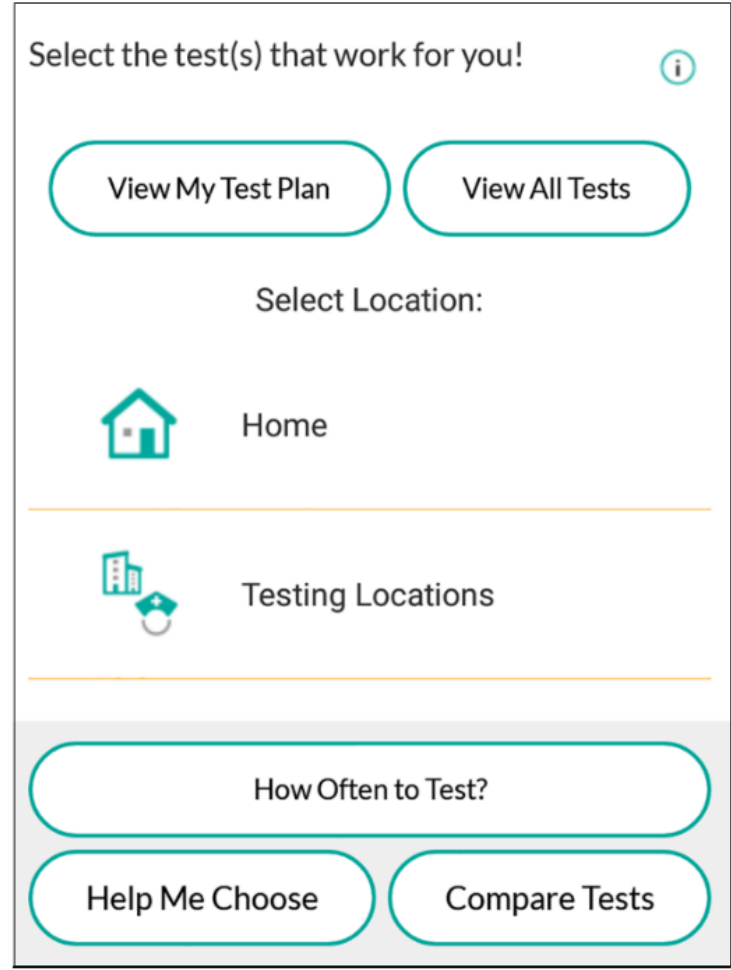

\section{Goal Setting and Environmental Influences}

The MyChoices app allows users to create an individually tailored HIV testing plan by having them compare and choose different options (eg, antigen-antibody testing to identify very recent infection vs rapid testing at home or in a clinic for convenience and quick answers) and provides answers to questions about HIV transmission behaviors and testing history (Figure 4).
After an HIV testing plan is developed, users have the option to customize the timing and content of testing reminders (eg, users can select from a list of phrases or create their own reminder to ensure privacy). Geofencing technology provides users with notifications to test if in the vicinity of a testing location (based on their GPS location) during the testing timeframe. 


\section{Data Collection}

Baseline assessments will be conducted at the enrolling clinical site using CASI, with all follow-up assessments at 3 and 6 months conducted off-site and through Web. At each major assessment, participants will complete a CASI-based assessment battery via a secure Web-based data entry system. Outcome domains and measures are described in Table 1.

Table 1. Outcomes and measures for MyChoices pilot randomized controlled trial.

\begin{tabular}{|c|c|}
\hline Domain & Description or scale \\
\hline \multicolumn{2}{|l|}{ Primary outcomes } \\
\hline App acceptability & System Usability Scale $[28,32]$ \\
\hline App feasibility & Proportion using the app $\geq 1$ time \\
\hline \multicolumn{2}{|l|}{ Secondary outcomes } \\
\hline HIV testing & Proportion testing at least once during study \\
\hline $\operatorname{PrEP}^{\mathrm{a}}$ uptake & Proportion of those with a behavioral indication for PrEP who are prescribed and utilize $\mathrm{F}$ \\
\hline \multicolumn{2}{|c|}{ Social cognitive theory model constructs outcomes } \\
\hline Self-regulation & Frequency of use of relevant app components, perceived HIV or STI ${ }^{\mathrm{b}}$ risk [33] \\
\hline Self-efficacy & HIV testing self-efficacy [34], PrEP use self-efficacy [35], condom use self-efficacy [36] \\
\hline Goal setting & Frequency of use of HIV testing plan \\
\hline Environmental influences & Frequency of use of reminders, frequency of testing due to geofencing technology \\
\hline
\end{tabular}

Covariates (based on ecosocial model) [37]

Individual

Demographics, socioeconomic posi- Age, race or ethnicity, student status, education, income, family structure, employment, insurance status tion

Sexual behavior (\# sex partners, condom use, partner selection)

Adapted from the AIDS Risk Behavior Assessment [38]

Drug use behavior (ie, alcohol, cocaine, meth)

Mental health (depression, anxiety) Personal Health Questionnaire Depression 8-Item Scale [40], Generalized Anxiety Disorder 7-Item

Trauma and abuse

Social

Social support

Peer norms for condom use

\section{Structural}

$\mathrm{SRV}^{\mathrm{c}}$ or city

Incarceration history

Structural discrimination

Stigma

\section{Other covariates}

Mobile phone and technology use

Mobile app use over the study period

HIV Negative Cascade Scale [41], sexual minority stress [42]

Startle, Physically Upset, Anger, and Numbness Posttraumatic Stress Scale [43]

Patient-Reported Outcomes Measurement Information System [44]

Questions regarding peer's use and perceptions for condoms [45,46]

Alcohol, Smoking, and Substance Involvement Screening Test [39]

Geographic location of study participant

Recent history and frequency

External homophobia [42], racism [47]

HIV-related, PrEP-related

Pew research technology use questionnaire $[48,49]$

Log in attempts, HIV testing and PrEP use, proportion complete HIV testing plans, proportion requesting HIV or STI home-test kits

HIV or STI testing history, PrEP awareness, barriers to PrEP uptake, PrEP adherence [50], barriers to PrEP use

${ }^{\text {a } P r E P: ~ p r e-e x p o s u r e ~ p r o p h y l a x i s . ~}$

${ }^{b}$ STI: sexually transmitted infection.

${ }^{\mathrm{c}} \mathrm{SRV}$ : subject recruitment venues. 


\section{Primary Outcome Measures}

To measure acceptability of the MyChoices app, we will use the SUS, a validated 10-item measure that assesses subjective usability of a system, or, in this case, an app [28]. It is scored from 0 to 100 , and a score of $\geq 50$ indicates that the app is acceptable [32]. To determine feasibility, we will use app analytics to determine whether at least $60 \%$ of individuals randomized to the intervention condition opened the My Choices app at least once after their initial introduction to the app by research staff. We will also assess the proportion of participants who complete their HIV testing plan (regardless of planned testing intervals) — a primary function of the app.

\section{Secondary Outcome Measures}

While this pilot study is not powered to examine the efficacy of behavioral outcomes, at each major assessment, participants will be asked to self-report on HIV testing in the previous 3-month interval. We will also assess self-report accuracy by obtaining medical release for HIV test results. Additionally, at each assessment, participants will self-report whether, in the past 3 months, they made and attended a clinic appointment for PrEP initiation, whether they were prescribed PrEP, and whether they utilized PrEP.

\section{Tertiary Outcome Measures}

All measures are outlined in detail in Table 1. In brief, congruent with our theoretical model, we will assess the following: (1) Self-regulation: perceived HIV and STI risk and transmission knowledge; (2) Self-efficacy: HIV testing, PrEP use, and condom use self-efficacy; and (3) Environmental influences: perceived utility of reminders for HIV testing. Each of these variables will mirror the content of the app, and we will adapt validated scales when available [51]. We will also assess individual (eg, sexual behavior and mental health), social (eg, social support), and structural (eg, incarceration and stigma) covariates. Finally, among those randomized to the MyChoices condition, we will use app analytic data to assess the frequency and duration of app use, content and functionalities most and least utilized, and requests for HIV or STI testing kits, condoms, and lube.

\section{Statistical Analyses}

The primary analyses will summarize acceptability (mean score on the SUS) and feasibility (participants utilizing the app at least once during follow-up) of the app, overall for the intervention arm, with asymptotic normal 95\% CIs. Point estimates for mean acceptability $\geq 50$ and for proportion accessing the app $>0.60$ will be considered the minimum criteria for acceptability and feasibility, consistent with industry standards [28,32].

The primary efficacy analysis will compare HIV testing (defined by the proportion that self-reports at least 1 HIV test result during follow-up) between the study arms at 3- and 6-month follow-ups. Group differences in self-reported PrEP-related appointments and documented HIV test results will also be examined at each time point. Moreover, group differences in measures related to the SCT model constructs (eg, HIV testing self-efficacy) will be assessed. The distribution of all variables will be assessed, and appropriate tests will be conducted (ie, parametric chi-square test vs nonparametric Fisher's exact test).

All analyses will use two-tailed tests of significance, with significance at alpha=.05. We will follow an intent-to-treat approach [52], analyzing participants in the study arm to which they were assigned. We will examine the equivalence of random assignment to groups with regards to key baseline characteristics, including sociodemographics, prior HIV testing patterns, and sexual risk-related variables. In the event that randomization does not work to balance these characteristics, we will assess whether baseline differences may account for differences in outcomes.

\section{Results}

Recruitment for the RCT began in October 2018, with study follow-up complete by June 2019.

\section{Discussion}

Advances in mobile phone technologies have enabled YMSM to have immediate access to broad social and sexual networks. The proposed project responds to the increasingly widespread use of mobile technology in the United States. MyChoices is a theory-driven app that was developed by our interdisciplinary team of HIV clinicians, epidemiologists, behavioral scientists, and app developers based on feedback from a diverse sample of YMSM and will be refined and tested using scientifically rigorous research methods. Therefore, to our knowledge, MyChoices will be one of the first comprehensive, theory-driven HIV prevention apps designed specifically for YMSM. We anticipate that MyChoices will increase HIV testing and linkage to prevention services because it is developmentally appropriate and meets YMSM where they are, in an environment that is familiar and discrete.

Anticipated limitations of this protocol include the self-reported outcomes for HIV testing and PrEP uptake. We will explore obtaining more objective measures of HIV testing and PrEP uptake, including obtaining photographs of test results and PrEP prescriptions. Relatedly, by answering questions about their HIV testing history at follow-up assessments, self-reported outcomes may be influenced that could bias the results. However, the follow-up survey covers a number of topics, including mental health, stigma, and sexual behaviors, and the HIV testing questions are limited. As a result, we do not anticipate substantial misclassification. There is also a risk that participants in the intervention group may discuss with or even show the MyChoices app to participants in the standard of care group (eg, if friends or partners both enroll in the study). To reduce the risk of contamination, study staff will remind the participants at baseline not to discuss the app with peers who might be in the study.

If MyChoices demonstrates acceptability and feasibility in this pilot study, a multicity, 3-arm RCT of this app and another youth-optimized app (LYNX) versus standard of care will be conducted through iTech. If shown to be efficacious, the MyChoices app could be a scalable technology-based solution, with the ability to reach YMSM across the United States, while 
maintaining a "geographically individualized" feel with app content integrated with local prevention and testing activities.

\section{Acknowledgments}

The authors would like to thank Adi Ferrara, MS, ELS, for help in the preparation of this manuscript.

\section{Conflicts of Interest}

None declared.

\section{References}

1. Centers for Disease Control and Prevention. 2016 Nov. HIV Surveillance Report: Diagnoses of HIV Infection in the United States and Dependent Areas, 2015 URL: https://www.cdc.gov/hiv/pdf/library/reports/surveillance/ cdc-hiv-surveillance-report-2015-vol-27.pdf [accessed 2018-11-04] [WebCite Cache ID 73fi1m6i9]

2. Centers for Disease Control and Prevention. 2015 Jul. Monitoring selected national HIV prevention and care objectives by using HIV surveillance data - United States and 6 dependent areas? 2013 URL: https://www.cdc.gov/hiv/pdf/library/reports/ surveillance/cdc-hiv-surveillancereport_vol20_no2.pdf [accessed 2018-11-04] [WebCite Cache ID 73fiKJpgd]

3. Wejnert C, Hess KL, Rose CE, Balaji A, Smith JC, Paz-Bailey G, NHBS Study Group. Age-Specific Race and Ethnicity Disparities in HIV Infection and Awareness Among Men Who Have Sex With Men--20 US Cities, 2008-2014. J Infect Dis 2016 Mar 01;213(5):776-783. [doi: 10.1093/infdis/jiv500] [Medline: 26486637]

4. Cohen MS, Chen YQ, McCauley M, Gamble T, Hosseinipour MC, Kumarasamy N, HPTN 052 Study Team. Prevention of HIV-1 infection with early antiretroviral therapy. N Engl J Med 2011 Aug 11;365(6):493-505 [FREE Full text] [doi: 10.1056/NEJMoa1105243] [Medline: 21767103]

5. Marks G, Crepaz N, Senterfitt JW, Janssen RS. Meta-Analysis of High-Risk Sexual Behavior in Persons Aware and Unaware They are Infected With HIV in the United States. JAIDS Journal of Acquired Immune Deficiency Syndromes 2005;39(4):446-453. [doi: 10.1097/01.qai.0000151079.33935.79]

6. Severe P, Jean Juste MA, Ambroise A, Eliacin L, Marchand C, Apollon S, et al. Early versus Standard Antiretroviral Therapy for HIV-Infected Adults in Haiti. N Engl J Med 2010 Jul 15;363(3):257-265. [doi: 10.1056/nejmoa0910370]

7. Grant RM, Lama JR, Anderson PL, McMahan V, Liu AY, Vargas L, iPrEx Study Team. Preexposure chemoprophylaxis for HIV prevention in men who have sex with men. N Engl J Med 2010 Dec 30;363(27):2587-2599 [FREE Full text] [doi: 10.1056/NEJMoa1011205] [Medline: 21091279]

8. McCormack S, Dunn DT, Desai M, Dolling DI, Gafos M, Gilson R, et al. Pre-exposure prophylaxis to prevent the acquisition of HIV-1 infection (PROUD): effectiveness results from the pilot phase of a pragmatic open-label randomised trial. Lancet 2016 Jan 02;387(10013):53-60 [FREE Full text] [doi: 10.1016/S0140-6736(15)00056-2] [Medline: 26364263]

9. Sanchez TH, Sineath RC, Kahle EM, Tregear SJ, Sullivan PS. The Annual American Men's Internet Survey of Behaviors of Men Who Have Sex With Men in the United States: Protocol and Key Indicators Report 2013. JMIR Public Health Surveill 2015;1(1):e3 [FREE Full text] [doi: 10.2196/publichealth.4314] [Medline: 27227126]

10. Krakower DS, Mimiaga MJ, Rosenberger JG, Novak DS, Mitty JA, White JM, et al. Limited Awareness and Low Immediate Uptake of Pre-Exposure Prophylaxis among Men Who Have Sex with Men Using an Internet Social Networking Site. PLoS One 2012;7(3):e33119 [FREE Full text] [doi: 10.1371/journal.pone.0033119] [Medline: 22470438]

11. Mayer K, Biello K, Novak D, Krakower D, Mimiaga M. PrEP uptake disparities in a diverse on-line sample of US men who have sex with men. 2017 Feb Presented at: Conference on Retroviruses and Opportunistic Infections; February 13-16, 2017; Seattle, Washington.

12. Arnett J. Emerging Adulthood: The Winding Road from the Late Teens Through the Twenties. New York, NY: Oxford University Press; 2014.

13. Gruber HE, Voneche JJ, editors. The essential Piaget. London: Routledge \& Kegan Paul PLC; 1977.

14. Anderson AM, Higgins MK, Ownby RL, Waldrop-Valverde D. Changes in neurocognition and adherence over six months in HIV-infected individuals with cocaine or heroin dependence. AIDS Care 2015;27(3):333-337 [FREE Full text] [doi: 10.1080/09540121.2014.985183] [Medline: 25484035]

15. Fjeldsoe BS, Marshall AL, Miller YD. Behavior change interventions delivered by mobile telephone short-message service. Am J Prev Med 2009 Feb;36(2):165-173. [doi: 10.1016/j.amepre.2008.09.040] [Medline: 19135907]

16. Blas MM, Alva IE, Carcamo CP, Cabello R, Goodreau SM, Kimball AM, et al. Effect of an online video-based intervention to increase HIV testing in men who have sex with men in Peru. PLoS One 2010 May 03;5(5):e10448 [FREE Full text] [doi: 10.1371/journal.pone.0010448] [Medline: 20454667]

17. Blas MM, Menacho LA, Alva IE, Cabello R, Orellana ER. Motivating men who have sex with men to get tested for HIV through the internet and mobile phones: a qualitative study. PLoS One 2013;8(1):e54012 [FREE Full text] [doi: 10.1371/journal.pone.0054012] [Medline: 23320116] 
18. Hirshfield S, Chiasson MA, Joseph H, Scheinmann R, Johnson WD, Remien RH, et al. An online randomized controlled trial evaluating HIV prevention digital media interventions for men who have sex with men. PLoS One 2012;7(10):e46252 [FREE Full text] [doi: 10.1371/journal.pone.0046252] [Medline: 23071551]

19. Ko N, Hsieh C, Wang M, Lee C, Chen C, Chung A, et al. Effects of Internet popular opinion leaders (iPOL) among Internet-using men who have sex with men. J Med Internet Res 2013 Feb 25;15(2):e40 [FREE Full text] [doi: 10.2196/jmir.2264] [Medline: 23439583]

20. Muessig KE, Pike EC, Legrand S, Hightow-Weidman LB. Mobile phone applications for the care and prevention of HIV and other sexually transmitted diseases: a review. J Med Internet Res 2013 Jan 04;15(1):e1 [FREE Full text] [doi: 10.2196/jmir.2301] [Medline: 23291245]

21. Sullivan PS, Driggers R, Stekler JD, Siegler A, Goldenberg T, McDougal SJ, et al. Usability and Acceptability of a Mobile Comprehensive HIV Prevention App for Men Who Have Sex With Men: A Pilot Study. JMIR Mhealth Uhealth 2017 Mar 09;5(3):e26 [FREE Full text] [doi: 10.2196/mhealth.7199] [Medline: 28279949]

22. Biello K, Coffey-Esquivel J, Hosek S, Belzer M, Sullivan P, Oleson S. Development of a mobile-based application to increase uptake of HIV testing among young US men who have sex with men. 2016 Jul Presented at: 2016 International AIDS Conference; July 18-22, 2016; Durban, South Africa.

23. Bandura A. The anatomy of stages of change. Am J Health Promot 1997;12(1):8-10. [doi: 10.4278/0890-1171-12.1.8] [Medline: 10170438]

24. Bandura A. Health promotion by social cognitive means. Health Educ Behav 2004 Apr;31(2):143-164. [doi: 10.1177/1090198104263660] [Medline: 15090118 ]

25. Milena ZD, Dainora G, Alin S. Qualitative Research Methods: A comparison between focus-group and in-depth interview. Annals of the University of Ordea, Economic Science Series 2008;17(4):1279-1283 [FREE Full text]

26. Kruger RA, Casey MA. Focus Groups: A Practical Guide For Applied Research. Thousand Oaks, CA: Sage Publications; 2015.

27. Carlsen B, Glenton C. What about N? A methodological study of sample-size reporting in focus group studies. BMC Med Res Methodol 2011 Mar 11;11:26 [FREE Full text] [doi: 10.1186/1471-2288-11-26] [Medline: 21396104]

28. Brooke J. SUS - A quick and dirty usability scale. In: Jordan PW, Thomas B, Weerdmeester BA, McClelland IL, editors. Usability evaluation in industry. London, GB: Taylor \& Francis; 1996:189-194.

29. Beebe J. Rapid Assessment Process. In: Kempf-Leonard K, editor. Encyclopedia of social measurement. Atlanta, GA: Elsevier; 2005:285-291.

30. Creswell J. Qualitative Inquiry and Research Design: Choosing among Five Approaches. Los Angeles, CA: Sage Publications; 2012.

31. Kernan WN, Viscoli CM, Makuch RW, Brass LM, Horwitz RI. Stratified randomization for clinical trials. J Clin Epidemiol 1999 Jan;52(1):19-26. [Medline: 9973070]

32. Bangor A, Kortum P, Miller J. Determining what individual SUS scores mean: Adding an adjective rating scale. Journal of Usability Studies 2009;4(3):114-123 [FREE Full text]

33. Napper LE, Fisher DG, Reynolds GL. Development of the perceived risk of HIV scale. AIDS Behav 2012 May;16(4):1075-1083 [FREE Full text] [doi: 10.1007/s10461-011-0003-2] [Medline: 21785873]

34. Jamil M, Guy RJ, Bavinton BR, Fairley C, Grulich A, Holt M, FORTH Investigator Group. HIV testing self-efficacy is associated with higher HIV testing frequency and perceived likelihood to self-test among gay and bisexual men. Sex Health 2017 Apr;14(2):170-178. [doi: 10.1071/SH16100] [Medline: 27883311]

35. Johnson MO, Neilands TB, Dilworth SE, Morin SF, Remien RH, Chesney MA. The role of self-efficacy in HIV treatment adherence: validation of the HIV Treatment Adherence Self-Efficacy Scale (HIV-ASES). J Behav Med 2007 Oct;30(5):359-370 [FREE Full text] [doi: 10.1007/s10865-007-9118-3] [Medline: 17588200]

36. Brafford LJ, Beck KH. Development and validation of a condom self-efficacy scale for college students. J Am Coll Health 1991 Mar;39(5):219-225. [doi: 10.1080/07448481.1991.9936238] [Medline: 1783705]

37. Krieger N. Epidemiology and the web of causation: has anyone seen the spider? Soc Sci Med 1994 Oct;39(7):887-903. [Medline: 7992123]

38. Donenberg GR, Emerson E, Bryant FB, Wilson H, Weber-Shifrin E. Understanding AIDS-risk behavior among adolescents in psychiatric care: links to psychopathology and peer relationships. J Am Acad Child Adolesc Psychiatry 2001 Jun;40(6):642-653 [FREE Full text] [doi: 10.1097/00004583-200106000-00008] [Medline: 11392341]

39. WHO ASSIST Working Group. The Alcohol, Smoking and Substance Involvement Screening Test (ASSIST): development, reliability and feasibility. Addiction 2002 Sep;97(9):1183-1194. [Medline: 12199834]

40. Kroenke K, Strine TW, Spitzer RL, Williams JBW, Berry JT, Mokdad AH. The PHQ-8 as a measure of current depression in the general population. J Affect Disord 2009 Apr;114(1-3):163-173. [doi: 10.1016/j.jad.2008.06.026] [Medline: 18752852]

41. Spitzer RL, Kroenke K, Williams JBW, Löwe B. A brief measure for assessing generalized anxiety disorder: the GAD-7. Arch Intern Med 2006 May 22;166(10):1092-1097. [doi: 10.1001/archinte.166.10.1092] [Medline: 16717171]

42. Hatzenbuehler ML, Nolen-Hoeksema S, Erickson SJ. Minority stress predictors of HIV risk behavior, substance use, and depressive symptoms: results from a prospective study of bereaved gay men. Health Psychol 2008 Jul;27(4):455-462. [doi: 10.1037/0278-6133.27.4.455] [Medline: 18643003] 
43. Meltzer-Brody S, Churchill E, Davidson JR. Derivation of the SPAN, a brief diagnostic screening test for post-traumatic stress disorder. Psychiatry Research 1999 Oct;88(1):63-70. [doi: 10.1016/s0165-1781(99)00070-0]

44. Cella D, Riley W, Stone A, Rothrock N, Reeve B, Yount S, PROMIS Cooperative Group. The Patient-Reported Outcomes Measurement Information System (PROMIS) developed and tested its first wave of adult self-reported health outcome item banks: 2005-2008. J Clin Epidemiol 2010 Nov;63(11):1179-1194 [FREE Full text] [doi: 10.1016/j.jclinepi.2010.04.011] [Medline: 20685078]

45. Catania JA, Osmond D, Stall RD, Pollack L, Paul JP, Blower S, et al. The continuing HIV epidemic among men who have sex with men. Am J Public Health 2001 Jun;91(6):907-914. [Medline: 11392933]

46. Latkin CA, Forman V, Knowlton A, Sherman S. Norms, social networks, and HIV-related risk behaviors among urban disadvantaged drug users. Social Science \& Medicine 2003 Feb;56(3):465-476. [doi: 10.1016/s0277-9536(02)00047-3]

47. Williams DR, Yan Y, Jackson JS, Anderson NB. Racial Differences in Physical and Mental Health: Socio-economic Status, Stress and Discrimination. J Health Psychol 1997 Jul;2(3):335-351. [doi: 10.1177/135910539700200305] [Medline: 22013026]

48. Zickuhr K, Smith A. Pew Research Center. 2012 Apr 13. Digital Differences URL: http://www.pewinternet.org/2012/04/ 13/digital-differences/ [accessed 2018-11-04] [WebCite Cache ID 73fmbzYmG]

49. Smith A. Pew Research Center. 2015 Apr 01. U.S. Smartphone Use in 2015 URL: http://www.pewinternet.org/2015/04/ 01/us-smartphone-use-in-2015/ [accessed 2018-11-04] [WebCite Cache ID 73fn2mS2h]

50. Lu M, Safren SA, Skolnik PR, Rogers WH, Coady W, Hardy H, et al. Optimal recall period and response task for self-reported HIV medication adherence. AIDS Behav 2008 Jan;12(1):86-94. [doi: 10.1007/s10461-007-9261-4] [Medline: 17577653]

51. Snead MC, O'Leary AM, Mandel MG, Kourtis AP, Wiener J, Jamieson DJ, et al. Relationship between social cognitive theory constructs and self-reported condom use: assessment of behaviour in a subgroup of the Safe in the City trial. BMJ Open 2014 Dec 30;4(12):e006093 [FREE Full text] [doi: 10.1136/bmjopen-2014-006093] [Medline: 25550295]

52. Detry MA, Lewis RJ. The intention-to-treat principle: how to assess the true effect of choosing a medical treatment. JAMA 2014 Jul 02;312(1):85-86. [doi: 10.1001/jama.2014.7523] [Medline: 25058221]

Abbreviations
CASI: computer-assisted self-interviewing
GPS: global positioning system
GIF: graphics interchange format
MSM: men who have sex with men
PrEP: pre-exposure prophylaxis
RCT: randomized controlled trial
SCT: social cognitive theory
SRV: subject recruitment venues
STI: sexually transmitted infection
SUS: System Usability Scale
YMSM: young men who have sex with men

Edited by A Pettifor, B Mustanski, I Holloway, S Allison, $R$ Schnall; submitted 04.04.18; peer-reviewed by H Cho, A Outlaw, KM Jackman; comments to author 22.05.18; revised version received 16.07.18; accepted 13.09.18; published 07.01.19

Please cite as:

Biello KB, Marrow E, Mimiaga MJ, Sullivan P, Hightow-Weidman L, Mayer KH

A Mobile-Based App (MyChoices) to Increase Uptake of HIV Testing and Pre-Exposure Prophylaxis by Young Men Who Have Sex With Men: Protocol for a Pilot Randomized Controlled Trial

JMIR Res Protoc 2019;8(1):e10694

URL: http://www.researchprotocols.org/2019/1/e10694/

doi: $10.2196 / 10694$

PMID: 30617042

CKatie B Biello, Elliot Marrow, Matthew J Mimiaga, Patrick Sullivan, Lisa Hightow-Weidman, Kenneth H Mayer. Originally published in JMIR Research Protocols (http://www.researchprotocols.org), 07.01.2019. This is an open-access article distributed under the terms of the Creative Commons Attribution License (https://creativecommons.org/licenses/by/4.0/), which permits unrestricted use, distribution, and reproduction in any medium, provided the original work, first published in JMIR Research Protocols, is properly cited. The complete bibliographic information, a link to the original publication on http://www.researchprotocols.org, as well as this copyright and license information must be included. 